\title{
Application of vibro-acoustic operational transfer path analysis
}

\author{
Vaitkus, Dovydas; Tcherniak, Dmitri; Brunskog, Jonas
}

\section{Published in:}

Applied Acoustics

Link to article, DOI:

10.1016/j.apacoust.2019.04.033

Publication date:

2019

Document Version

Early version, also known as pre-print

Link back to DTU Orbit

Citation (APA):

Vaitkus, D., Tcherniak, D., \& Brunskog, J. (2019). Application of vibro-acoustic operational transfer path analysis. Applied Acoustics, 154, 201-212. https://doi.org/10.1016/j.apacoust.2019.04.033

\section{General rights}

Copyright and moral rights for the publications made accessible in the public portal are retained by the authors and/or other copyright owners and it is a condition of accessing publications that users recognise and abide by the legal requirements associated with these rights.

- Users may download and print one copy of any publication from the public portal for the purpose of private study or research.

- You may not further distribute the material or use it for any profit-making activity or commercial gain

- You may freely distribute the URL identifying the publication in the public portal

If you believe that this document breaches copyright please contact us providing details, and we will remove access to the work immediately and investigate your claim. 


\title{
Application of Vibro-Acoustic Operational Transfer Path Analysis
}

\author{
Dovydas Vaitkus $^{\mathrm{a}, *}$, Dmitri Tcherniak ${ }^{\mathrm{b}}$, Jonas Brunskog ${ }^{\mathrm{a}}$ \\ ${ }^{a}$ Department of Electrical Engineering, Technical University of Denmark, Lyngby, Denmark \\ ${ }^{b}$ Brüel \&s Kjar Sound and Vibration Measurement $A / S$, \\ Narum, Denmark
}

\begin{abstract}
Transfer Path Analysis (TPA) is widely used to identify individual path contributions and to solve Noise, Vibration and Harshness (NVH) issues in different industries. However, the complexity and technical burden of the conventional TPA calls for alternative, faster techniques. One such alternative is the Operational Transfer Path Analysis (OTPA), which uses only operational data to estimate the contributions. However, this approach has its own limitations; in some situations, the OTPA results may be affected, which can lead to the wrong engineering decisions. This paper presents an alternative formulation of OTPA, in application to structure-borne noise: Operational Transfer Path Analysis - Difference (OTPA-D). The theoretical considerations and analytical model showed that OTPA-D solves one of the crucial issue regarding the OTPA technique. The paper provides theoretical considerations behind OTPA-D; also, a laboratory model is set up to compare the results of three versions of OTPA with the results of TPA. It is shown that two experimental OTPA versions matched TPA better compared to the traditional OTPA method.
\end{abstract}

Keywords:

Transfer Path Analysis, Operational Transfer Path Analysis, Operational Transfer Path Analysis-Difference, Frequency Response Function,

\footnotetext{
* Corresponding author

Email addresses: dovydasvaitkus1992@gmail.com (Dovydas Vaitkus), Dmitri.Tcherniak@bksv.com (Dmitri Tcherniak), jbr@elektro.dtu.dk (Jonas Brunskog)
} 
Transmissibility Matrix, Vibration Transmission, Structure-Borne sound, Individual Path Contributions

\section{Introduction}

In daily life, people using mechanisms are surrounded by the sounds produced by a variety of sources. However, not all sounds can be perceived as pleasant ones. These unwanted sounds is noise, often generated by an engi5 neering structure, for example, the engine of a vehicle. The noise, caused by the engine, propagates to the position of interest through different paths, where each of them contributes differently. The path contributions cannot be measured directly; hence Transfer Path Analysis (TPA), introduced some decades ago 1], provided two experimental techniques for estimating the contributions indirectly. The first of the two, the mount-stiffness method can be only applied to structure-borne noise, whilst the second, which is often referred to as a matrix inversion method, can be applied to both structure-borne and air-borne problems. To estimate strengths of the noise sources and the sensitivity of the paths, TPA requires that the system is separated into two subsystems: active and passive, and Frequency Response Functions (FRFs) are measured between many points of the structure. This makes the application of TPA very time consuming. In order to reduce the required workload, a number of methods were introduced during the last two decades. One of them is Operational Transfer Path Analysis (OTPA), which is based on the Transmissibility Matrix (TM).

${ }_{20}$ The OTPA method does not require any FRF measurement, hence separation of the assembly into active and passive subsystems, as it is only based on operational measurements. However, the OTPA method has some disadvantages that are listed in references [2, 3, 4. These are the effect of neglected paths, cross-coupling, and the mathematical issues behind the TM estimation. Operational Path Analysis with eXogeneous inputs (OPAX) [5] claims to solve some of the above-mentioned problems via path modelling and finding the parameters of the model from operational data. The recent introduction of blocked forces 
[6, 7] and its application to TPA demonstrated that correct contributions can be obtained without disassembling the system into active and passive parts, which also allowed a significant reduction of the time necessary to conduct conventional TPA. An overview of existing methods addressing finding contributions from different sources and paths can be found in [1]. Nowadays, as electrical and hybrid vehicles are coming to the market, customers may experience new types of high frequency noise, which explains a renewed interest in the field [8].

In [9, a variation of OTPA, only suited for structure-borne noise, was introduced. Similar to the OTPA method, this approach uses the transmissibility matrix to estimate the individual path contributions, therefore the procedure is significantly faster than the conventional TPA. The difference is that this method uses mounts' deformation to estimate the contributions. As the defor-

40 mations are calculated as the difference between the responses measured at the active and the passive sides, the technique is referred to as Operational Transfer Path Analysis - Difference (OTPA-D).

The purpose of this paper is to investigate the OTPA-D method experimentally and to derive conclusions based on the obtained results. The paper starts 45 by briefly presenting different methods: TPA, OTPA, and OTPA-D, followed by the required measurement procedures in the analytical and experimental models. The analytical and experimental results are presented and discussed in the following sections.

\section{Method}

\subsection{The TPA method}

The individual path contributions using the TPA method [1, 10] are synthesized from the FRF, which describes the system of interest, and the operational forces acting in the system:

$$
\{Y\}=\left[H_{y f}\right]\{F\},
$$

representing an input-output relationship, where $\{F\}$ is the vector of operational input forces, $\left[H_{y f}\right]$ is the FRF matrix of the passive subsystem, and $\{Y\}$ is the 
vector of the responses. The FRF matrix of the system is usually determined experimentally. The crucial parameter that is used to synthesize individual path contributions is the operational input forces $\{F\}$. The forces can be determined by one of the following techniques:

- Using a force transducer - requires mounting a force transducer at the interface, between the active and passive subsystems, and directly measuring the force. The drawback of this method is that it requires modifying the mounting, which is often not feasible in practice.

- Mount stiffness method - uses mount deformations to estimate operational forces by applying Hook's law,

$$
\{F\}=\lceil K\rfloor\{\Delta X\}
$$

where $\lceil K\rfloor$ is the diagonal matrix of mounts' stiffnesses, and $\{\Delta X\}$ is the vector of mounts' deformations [1. The drawback is that the mount stiffness data often is neither available nor very accurate. The mount stiffness can be estimated experimentally, however, it can become quite complicated procedure, since it depends on various parameters [11].

- Matrix inversion method - is the most used technique [12, and uses the responses from addition accelerometers (indicators) $\{V\}$, similar to eq. (1), $\{V\}=\left[H_{v f}\right]\{F\}$. These so-called indicators are mounted close to the interfaces to get the best estimation. By inverting $\left[H_{v f}\right]$ matrix, operational forces can be found [12,

$$
\{F\}=\left[H_{v f}\right]^{+}\{V\}
$$

where + indicates a Moore-Penrose inversion [13. The main issue with matrix inversion is that it requires a quite significant amount of indicators responses in order to avoid ill-conditioning. The rule of thumb is to have at least two times more responses than the number of paths [1, 14].

From the FRF matrix and operational forces data, the individual contributions can be synthesized. For example, let us consider a very simple system 
having two paths with operational forces $F_{1}$ and $F_{2}$ acting at the interfaces. The paths are characterized by FRFs: $H_{11}$ and $H_{12}$, for one receiver. Then the individual contributions for the two paths are determined as:

$$
C_{11}=H_{11} F_{1}, \quad C_{12}=H_{12} F_{2},
$$

where $C_{11}$ and $C_{12}$ are the individual contributions. The summation of these two contributions results in the total response,

$$
Y_{1}=C_{11}+C_{12}
$$

In an ideal situation, the sum of individual contribution coincides with the measured data response $Y_{1}$. In practice, eq. (5) can be used to verify if all paths are included. If some of the paths are not covered, then the sum of individual contributions becomes different from the measured responses 2 .

In this study, the contributions estimated by the conventional TPA method are used as a baseline for comparison with other methods. TPA is referred to as Exact contributions later in the paper.

\subsection{The OTPA method}

OTPA uses a TM technique to estimate the individual path contributions. This can be shown by inserting eq. (3) into (1), which yields

$$
\{Y\}=\left[H_{y f}\right]\left[H_{v f}\right]^{+}\{V\}=\left[T_{y v}\right]\{V\} .
$$

The responses in eq. (6) are linked through the transmissibility matrix $\left[T_{y v}\right]$ that can be determined from operational measurements, by inverting the indicator responses [15],

$$
\left[T_{y v}\right]=\left[\{Y\}_{1} \ldots\{Y\}_{M}\right]\left[\{V\}_{1} \ldots\{V\}_{M}\right]^{+},
$$

where $M$ is the number of operational conditions, which have to be higher than the number of paths to avoid the errors regarding the ill-conditioning [1].

The individual path contribution for OTPA is determined in a similar way as for TPA. By taking the same example, where two paths and one receiver are 
considered, the individual contributions are determined,

$$
S_{11}=T_{11} V_{1}, \quad S_{12}=T_{12} V_{2}
$$

By summing the individual contributions the total response is achieved (9), which coincides with measured data,

$$
Y_{1}=S_{11}+S_{12}
$$

It was demonstrated [2] that unlike the TPA method, this summation is always equal to the response $Y_{1}$. Thus if one or several paths are neglected, the measured and summed data will still coincide, and the missing paths will not be detected. In addition to that, the individual contribution estimated with OTPA method faces two other issues: effects of cross-coupling and errors in transmissibility estimation [3, 4]. Due to these, individual contributions estimated by OTPA method are somewhat different from the ones estimated with TPA, thus, $S_{i j} \neq C_{i j}$. To demonstrate this, consider a simple system with two forces $\left\{x_{1}, x_{2}\right\}$, two indicators $\left\{v_{1}, v_{2}\right\}$, mounted on the passive side of the structure and one receiver $\left\{y_{1}\right\}$, similar to the one presented in [16]. The FRF matrices describing the relationship between sources and receiver, and between sources and indicators are $\left[H_{y x}\right],\left[H_{v x}\right]$,

$$
\left[H_{y x}\right]=\left\{a_{11} a_{12}\right\},\left[H_{v x}\right]=\left[\begin{array}{cc}
h_{11} & \varepsilon h_{12} \\
\varepsilon h_{21} & h_{22}
\end{array}\right]
$$

where $\varepsilon$ is a bookkeeping device, allowing us to follow the cross-coupling terms in $\left[H_{v x}\right]$. Following the OTPA method, the indicator accelerometers shall be placed close to the paths of interest (or around the source of interest, in air-borne noise case). In the considered case, the indicator accelerometer $v_{1}$ is placed close to the point where force $x_{1}$ is acting, and the indicator $v_{2}$ is placed near the acting point of force $x_{2}$. According to this consideration, one can generally assume that the diagonal terms of $\left[H_{v x}\right]$ are greater than the cross-diagonal ones and therefore $\varepsilon$ is small: $\varepsilon<1$. The TPA contributions for this case are

$$
y_{1}=a_{11} x_{1}+a_{12} x_{2}=C_{11}+C_{12} .
$$


To compute OTPA contributions (8), one needs the transmissibility matrix, which can be estimated from operating measurements via (7) or from definition (6): $\left[T_{y v}\right]=\left[H_{y x}\right]\left[H_{v x}\right]^{-1}$. For a simple $2 x 2$ case,

$$
\left[H_{v x}\right]^{-1}=\frac{1}{\operatorname{det}\left(\left[H_{v x}\right]\right)}\left[\begin{array}{lr}
h_{22} & -\varepsilon h_{12} \\
-\varepsilon h_{21} & h_{11}
\end{array}\right]
$$

where determinant is

$$
\operatorname{det}\left(\left[H_{v x}\right]\right)=h_{11} h_{22}-\varepsilon^{2} h_{12} h_{21} .
$$

Substituting this into $(12)$, and further into (8), and expanding into a Taylor series yields

$$
\begin{aligned}
& S_{11}=a_{11} x_{1}+\varepsilon\left(\frac{a_{11} h_{12} x_{2}}{h_{11}}-\frac{a_{12} h_{21} x_{1}}{h_{22}}\right)+O\left(\varepsilon^{2}\right) \\
& S_{12}=a_{12} x_{2}+\varepsilon\left(\frac{a_{12} h_{21} x_{1}}{h_{22}}-\frac{a_{11} h_{12} x_{2}}{h_{11}}\right)+O\left(\varepsilon^{2}\right)
\end{aligned}
$$

The first terms in the expressions can be identified as the TPA contributions (11), thus

$$
\begin{aligned}
& S_{11}=a_{11} x_{1}+O(\varepsilon)=C_{11}+O(\varepsilon), \\
& S_{12}=a_{12} x_{2}+O(\varepsilon)=C_{12}+O(\varepsilon) .
\end{aligned}
$$

From (15), it can be noted that $S_{11}=C_{11}$ and $S_{12}=C_{12}$ when $\varepsilon=0$, i.e. there is no cross-talk, in other words, the indicator $v_{1}$ does not pick up any energy from the force $x_{2}$ and indicator $v_{2}$ does not pick up anything from $x_{1}$, which is a very seldom case in reality. If $\varepsilon \neq 0$, the contributions provided by the OTPA method are generally wrong. The error depends on many parameters and its estimation can be hardly generalized. It is interesting to note that summing up $S_{11}$ and $S_{12}$, the terms in brackets cancel out, resulting in

$$
S_{11}+S_{12}=C_{11}+C_{12}+O\left(\varepsilon^{2}\right),
$$

80 in other words, the sum of OTPA contributions is the same as in TPA, which agrees with the observation in $[3]$.

In the presented example, the indicators were mounted on the passive side of the system. In [17], it was suggested to place the indicators on the active side 
of the mounts, as close as possible to the mounts. The variants are referred to as Method 1 and Method 2, respectively, later in the paper.

\subsection{The OTPA-D method}

Study [9] suggested another approach to determine the individual path contribution, using a concept very similar to OTPA.

According to eq. (2), the operational forces can be determined from mountstiffness and mount-deformation data. Substituting eq. (2) into (1) yields,

$$
\{Y\}=\left[H_{y f}\right]\lceil K\rfloor\{\Delta X\}
$$

showing the causal relationship between the input $\{\Delta X\}$ and output $\{Y\}$, similarly as for the TPA method. $\{\Delta X\}$ corresponds to the difference between the indicators responses measured at the active and the passive parts of the system: $\{\Delta X\}=\left\{X_{a}\right\}-\left\{X_{p}\right\}$, therefore the method is referred to as Operational Transfer Path Analysis - Difference (OTPA-D). In addition to that, (17) links the receivers responses $\{Y\}$ with the deformation responses $\{\Delta X\}[9$, which according to the transmissibility concept, can be expressed as the new transmissibility matrix

$$
\left[T_{y v}^{*}\right]=\left[H_{y f}\right]\lceil K\rfloor .
$$

Thus, the eq. 17) can be expressed as

$$
\{Y\}=\left[T_{y v}^{*}\right]\{\Delta X\}
$$

Applying the OTPA concept, the TM can be estimated from operational mea-

surements, making use of eq. (7), where instead of indicators signals $\{V\}$, the differences $\{\Delta X\}$ are used.

Previously it was shown that individual contributions $S_{i j}=C_{i j}$ if $\left[H_{v x}\right]$ matrix is diagonal (15). Here, this condition is fulfilled as $\lceil K\rfloor$ is diagonal and so is $\left[T_{y v}^{*}\right]$. This shows that TM for OTPA-D is not affected by the crosscoupling effect, which is the most criticized issue regarding the OTPA method [3, 16]. Considering the example with two paths and one receiver, the individual 
contributions can be determined from

$$
S_{11}^{*}=T_{11}^{*} \Delta X_{1}, \quad S_{12}^{*}=T_{12}^{*} \Delta X_{2}
$$

Now the individual contributions estimated with OTPA-D are the same as TPA, thus $S_{11}^{*}=C_{11}$ and $S_{12}^{*}=C_{12}$.

The OTPA-D method requires twice the amount of accelerometers compared with the OTPA method, which is one of the drawbacks. Also, OTPA-D inherits from OTPA the issues regarding the neglected paths and transmissibility estimation [2, 4]. The OTPA-D method will be referred to as Method 3 in the following sections.

\section{Procedure}

\subsection{Analytical Model}

To compare the TPA, OTPA and OTPA-D methods, a simple analytical five degrees of freedom (DoF) system, representing an engine mounted on a chassis through two mounts is considered [9]. The mass $m_{0}$ and rotational inertia $I_{0}$ represent the engine, while masses: $m_{1}, m_{2}, m_{3}$ represent the chassis as presented in figure 1. (Note that the engine is free to rotate.)

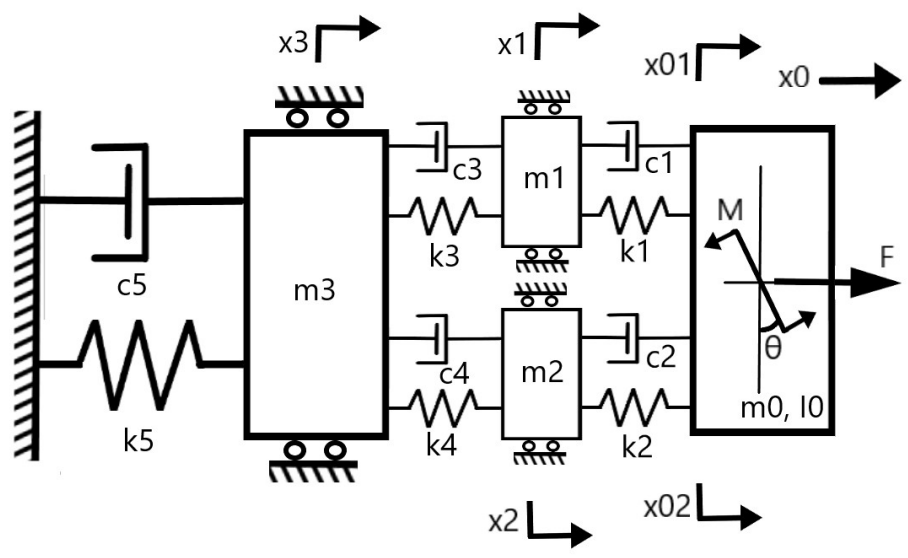

Figure 1: Engine mounting representation 
All the numerical values for the components are presented in tables 1 and 2. Excitation force $F^{(e x t)}$ simulates displacements $\left(x_{01}, x_{02}, x_{1}, x_{2}, x_{3}\right)$ which are used to identify operational forces and individual contributions. Test forces $F^{(1)}$ and $F^{(2)}$ are to generate transmissibility matrices for OTPA and OTPA-D methods.

\begin{tabular}{c|ccccc}
$\begin{array}{c}\text { Masses }[\mathrm{kg}] \text { \& Rotational } \\
\text { Inertia }\left[\mathrm{kg} \mathrm{m}^{2}\right]\end{array}$ & $m_{0}=100$ & $I_{0}=6.7$ & $m_{1}=20$ & $m_{2}=30$ & $m_{3}=800$ \\
\hline Stiffness $[\mathrm{N} / \mathrm{mm}]$ & $k_{1}=50$ & $k_{2}=40$ & $k_{3}=120$ & $k_{4}=160$ & $k_{5}=2$ \\
\hline Damping $[\mathrm{kg} / \mathrm{s}]$ & $c_{1}=5$ & $c_{2}=4$ & $c_{3}=12$ & $c_{4}=16$ & $c_{5}=0.2$
\end{tabular}

Table 1: Parameter values for the analytical model

\begin{tabular}{c|c} 
Excitation & $\{F\}^{(e x t)}=\{0.5 N, 0.5 N m\}^{T} \sin (\omega t)$ \\
\hline Test 1 & $\{F\}^{(1)}=\{0 N, 1 N m\}^{T} \sin (\omega t)$ \\
\hline Test 2 & $\{F\}^{(2)}=\{1 N, 0.5 N m\}^{T} \sin (\omega t)$
\end{tabular}

Table 2: Excitation forces for the analytical model

\subsubsection{TPA}

To determine individual contributions using the TPA method, the system has to be separated into active and passive, figure 2

The FRF matrix can be determined by exciting the passive part for each path individually: first $\left\{F_{1}\right\}=\{1,0,0\}^{T}$ and second $\left\{F_{2}\right\}=\{0,1,0\}^{T}$. A total of six FRFs are obtained, three for each path $\left(x_{1}, x_{2}, x_{3}\right)$.

Another step is to determine the operational forces. For such a simple system, the operational forces can be estimated using the mount stiffness method, which uses Hook's law (2) to determine the forces acting on the subsystems:

$$
F_{01}=\left(k_{1}+i \omega c_{1}\right)\left(x_{01}-x_{1}\right), \quad F_{02}=\left(k_{2}+i \omega c_{2}\right)\left(x_{02}-x_{2}\right) .
$$

Alternatively, the matrix inversion method can be used. 


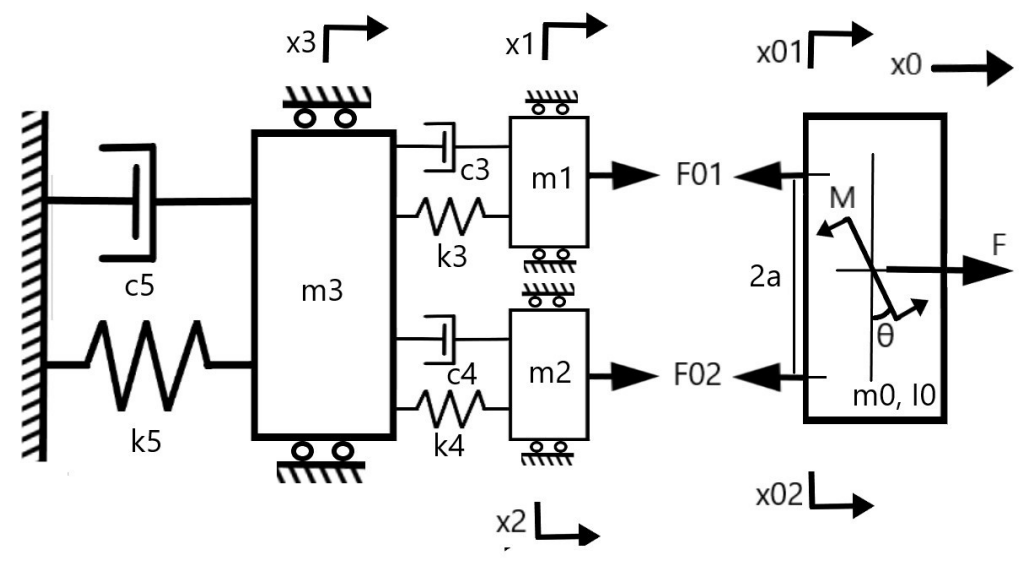

Figure 2: System separation: passive part on the left, active on the right

Applying the Excitation forces, that are presented in table 2, to the full system, operational displacements, $x_{1}, x_{2}, x_{01}, x_{02}$, can be obtained. From here the operational forces, $F_{01}$ and $F_{02}$, can be determined (21).

Finally, the individual path contributions can be synthesized by multiplying the estimated FRFs of the passive subsystem, $H_{i j}$, together with corresponding operational forces,

$$
C_{1}=H_{13} F_{01}, \quad C_{2}=H_{23} F_{02}
$$

\subsubsection{OTPA \& OTPA-D}

To estimate the individual path contribution for the three OTPA-based methods, the TMs have to be determined first. The procedure to determine TMs for all three methods is very similar. For two structure-borne paths, all three approaches require at least two different operational conditions that are presented in table 2

The first step is to apply both Test forces, $\{F\}^{(1)}$ and $\{F\}^{(2)}$, to the full system, and compute the responses. The TMs for three OTPA-based methods can be determined, according to eq. (7)

$$
\{T\}=\{Y\}[V]^{-1},
$$

where the difference is the indicators responses $\{V\}$ : 
- Method 1 - indicator responses at the passive subsystem: $\left\{V_{p}\right\}=\left\{\begin{array}{l}x_{1} \\ x_{2}\end{array}\right\}$.

- Method 2 - indicator responses at the active part: $\left\{V_{a}\right\}=\left\{\begin{array}{l}x_{01} \\ x_{02}\end{array}\right\}$.

- Method 3 - the OTPA-D method: $\{\Delta V\}=\left\{\begin{array}{l}x_{01}-x_{1} \\ x_{01}-x_{2}\end{array}\right\}$.

After the TM is estimated, the individual path contribution for all three methods are determined, according to (8) for Method $\mathbf{1}$ and 2, and according to 20 for Method 3, where the indicators responses $\{V\}$ are measured on the Excitation signal: $\{F\}^{(e x t)}$.

All three methods use the same principle in estimating individual contributions and are based on operational measurement data. The only difference between these three is that Method 3 determines TM and individual contributions from the difference of two responses, while Methods $1 \& 2$ are not.

The results from analytical model for Path 1 and Path 2 are presented in figures $3 \mathrm{a}$ and $3 \mathrm{~b}$ respectively.

From the analytical results, it can be observed that OTPA-D, which is referred to as Method 3, perfectly matches TPA, while OTPA: Method 1 and Method 2, match TPA only at certain frequency ranges. It was observed that OTPA methods match TPA rather well in the range where cross-talk between the paths is the lowest.

\subsection{Experimental Setup}

To validate all three OTPA-based methods, an experimental setup was built, figure $4 \mathrm{a}$.

Similar to the analytical model, the setup contains four parts, which are marked in figure $4 \mathrm{a}$

1. Active part - represents the engine. The vibrations in the active part are generated by three shakers, which are presented in figure $4 \mathrm{~b}$. 


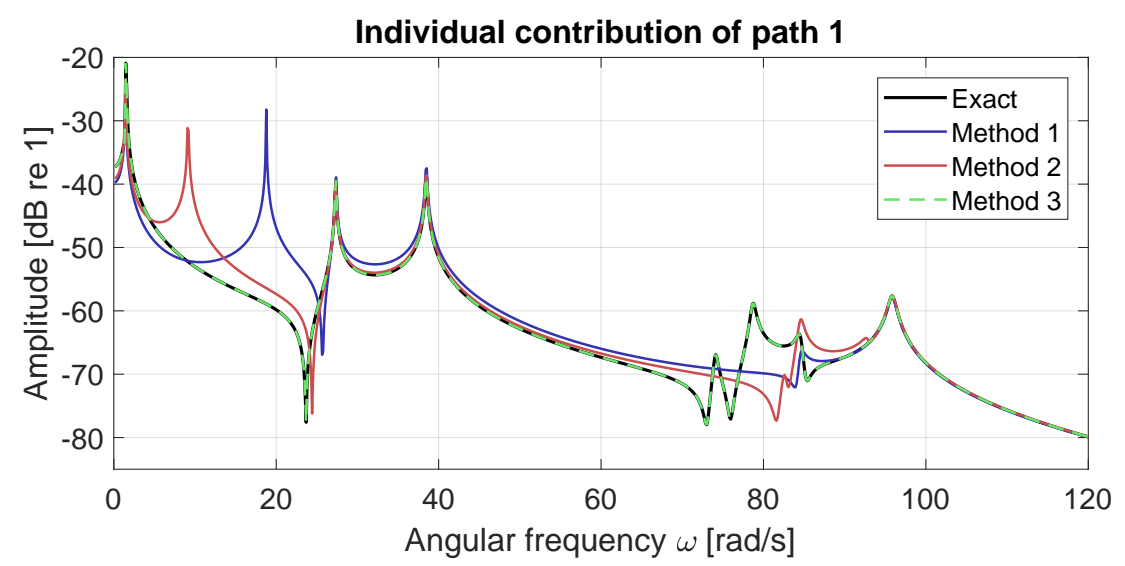

(a)

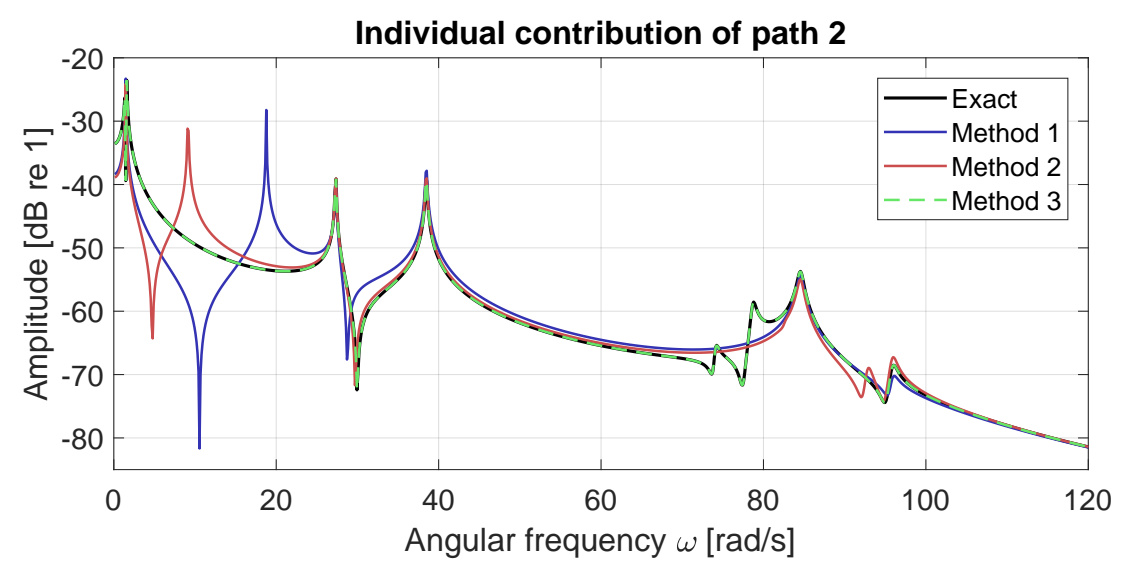

(b)

Figure 3: Individual Contributions, analytical model

2. Passive part - represents the chassis of the vehicle.

3. Engine mounts (figure 4c)

4. Suspension (figure $4 \mathrm{~d}$ )

The receivers that are used during the experiments are labelled accordingly: $R_{3}$ and $R_{4}$. Below each engine mount, an additional force transducer is mounted to validate matrix inversion results in the transverse direction.

The experimental setup contains nine structure-borne paths: three engine mounts where each moves in three directions $(X, Y, Z)$. The rotational DoFs are 


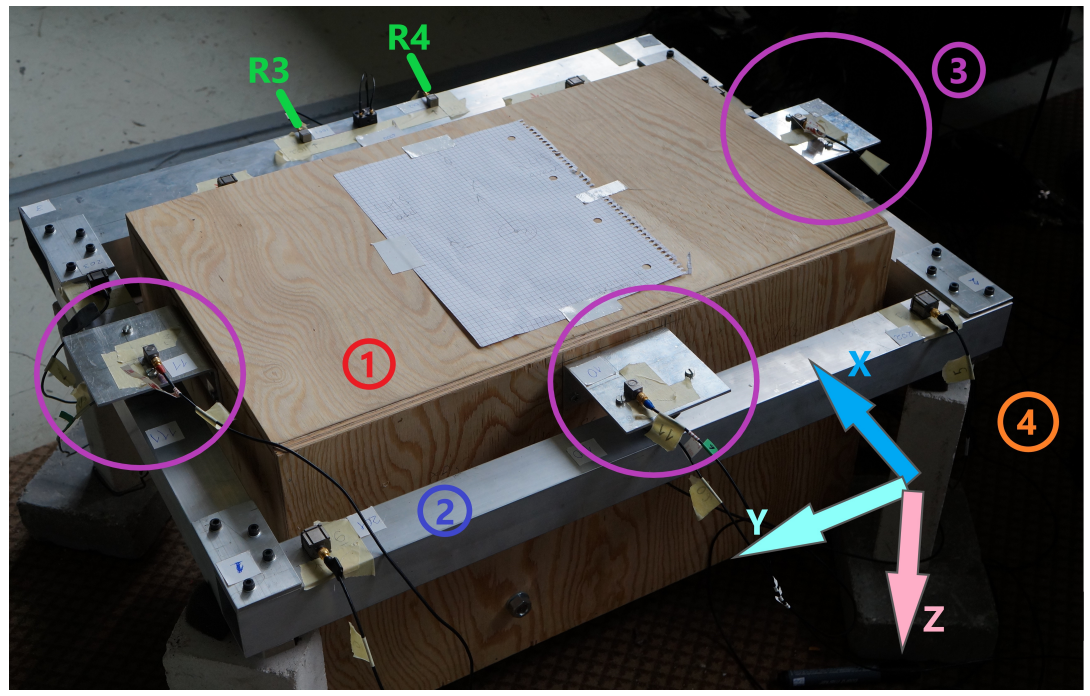

(a) Experimental setup

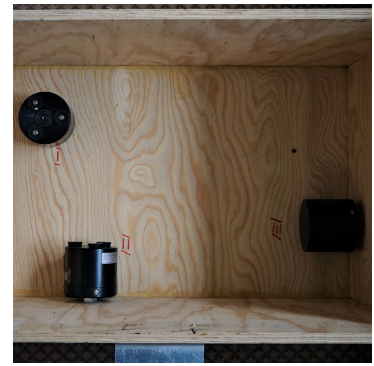

(b) Shakers

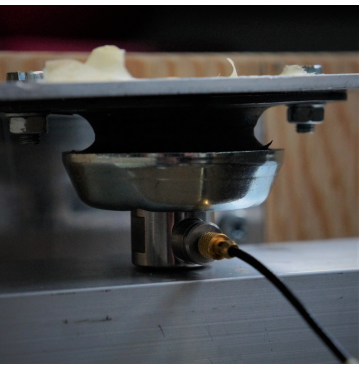

(c) Engine mount, force transducer

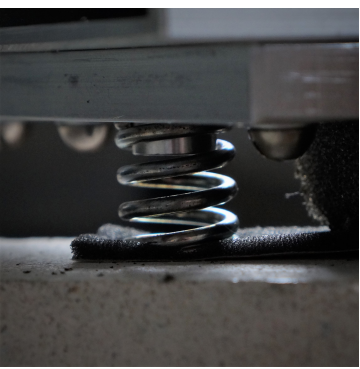

(d) Suspension

Figure 4

assumed to be minimal and therefore neglected. The procedure to estimate individual contribution with TPA and OTPA-based techniques are rather similar to the analytical model.

The signals that are used to estimate individual contributions and TM are 165 presented in table 3. 


\begin{tabular}{c|c|c} 
Excitation & Sine sweep: $1-1600 \mathrm{~Hz}$ & Used to find contributions \\
\hline Test & Random white noise: $1-1600 \mathrm{~Hz}$ & Used to calculate TMs
\end{tabular}

Table 3: Experimental excitation signals

\subsubsection{TPA}

To estimate the individual contributions using TPA method, the engine together with three engine mounts have to be dismounted before measuring FRFs. The FRFs matrix is measured by exciting each path with a modal hammer and measuring the responses at all the receivers and indicators positions. As an example, a few elements of the measured FRF matrix, when excited at path 3 , are presented in figure 5 .

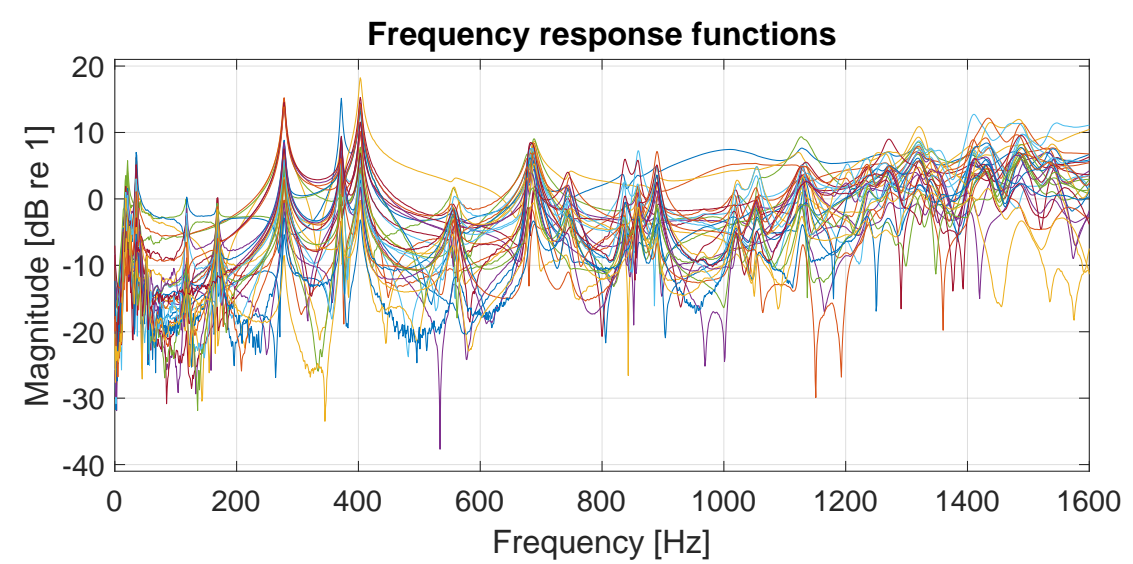

Figure 5: Element of FRF matrix, when excited at path 3

The operational forces are estimated using the matrix inversion method, eq. (3). In addition to that, from figure $4 \mathrm{c}$ it can be observed that additional force transducers, for each path, are mounted. These are used to validate the matrix inversion method in the transverse direction. To estimate operational forces using the matrix inversion method, the FRF matrix containing indicators responses, $\left[H_{v f}\right]$, have to be inverted. During the inversion, the lowest singular values, that are often caused by noise, are neglected. Then the inverted FRF matrix and the indicators responses are multiplied, resulting in operational forces. 
The estimated forces are compared with the measured ones in the same axis as presented in figure 6. It can be observed that the synthesized forces match the
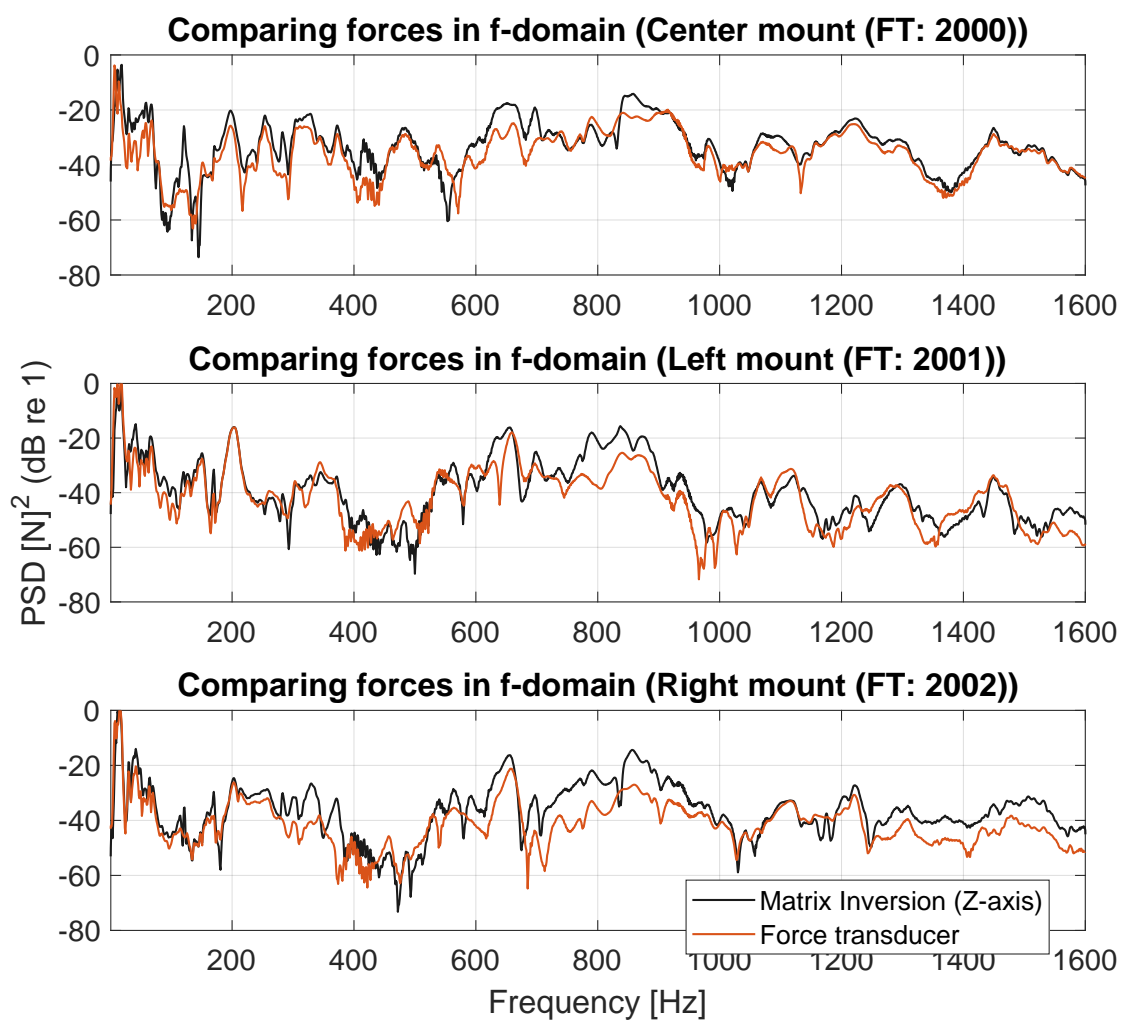

Figure 6: Estimated and measured forces

actual ones rather well. There is slight overdetermination in the Right mount (FT:2002).

The individual contributions for TPA method are now determined by multiplying the FRF matrix containing receivers' responses together with corresponding operational forces,

$$
C_{i j}=H_{i j} F_{j}, \quad Y_{i}=\sum_{j} C_{i j} .
$$

As mentioned previously, the sum of individual contributions $C_{i j}$ can be compared with the corresponding response $Y_{i}$ at the receiver position to validate the method. The measured response of receiver $R_{3}$ is compared with the sum 
of synthesized contributions in figure 7

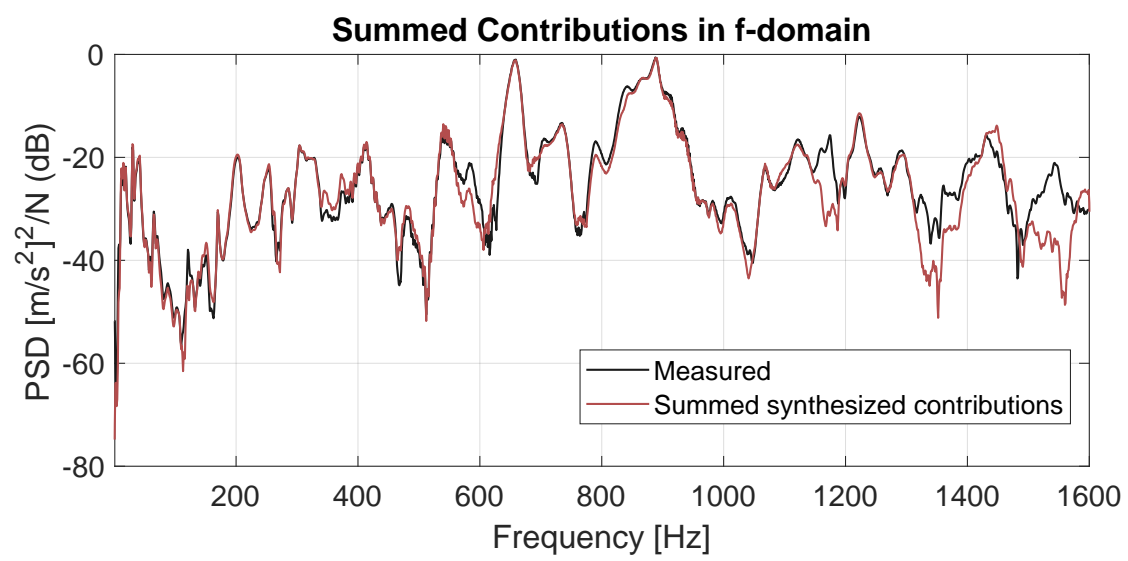

Figure 7: Measured receiver response versus summed contributions

The measured response $Y_{3}$ matched the sum of synthesized contributions

$\sum_{j} C_{3 j}$ quite well, meaning the TPA results can be trusted for further investigation.

\subsubsection{OTPA \& OTPA-D}

Regarding the OTPA-based methods, the first step is to estimate the TM, which requires the number of different operational conditions to be higher than the number of paths. The TM is determined using the $H_{s}$ estimator [18, which is based on singular value decomposition of the auto-power matrix $\left[G_{y v y v}\right]$ of the vector containing receiver $y$ and indicators $v$,

$$
\left[G_{y v y v}\right]=\left[\begin{array}{ll}
G_{y y} & G_{y v} \\
G_{v y} & G_{v v}
\end{array}\right]=\left[\begin{array}{ll}
U_{y} & V_{y} \\
U_{v} & V_{v}
\end{array}\right]\left[\begin{array}{ll}
\lambda_{N} & 0 \\
0 & \lambda_{M}
\end{array}\right]\left[\begin{array}{ll}
U_{y} & V_{y} \\
U_{v} & V_{v}
\end{array}\right]^{H}
$$

where $\lambda_{M}$ represents total amount of singular values. The lowest singular values are considered to be caused by noise and can be rejected [19] by limiting the inner matrix to the size of $\lambda_{N}$ [18. The transmissibility matrix is estimated from

$$
\left[T_{y v}\right]=\left[U_{y}\right]\left[U_{v}\right]^{+},
$$

using the highest singular values. The elements of the estimated TMs for receiver $R_{3}$, path 3 for each OTPA-based method are presented in figure 8 


\section{Transmissibility Matrices}

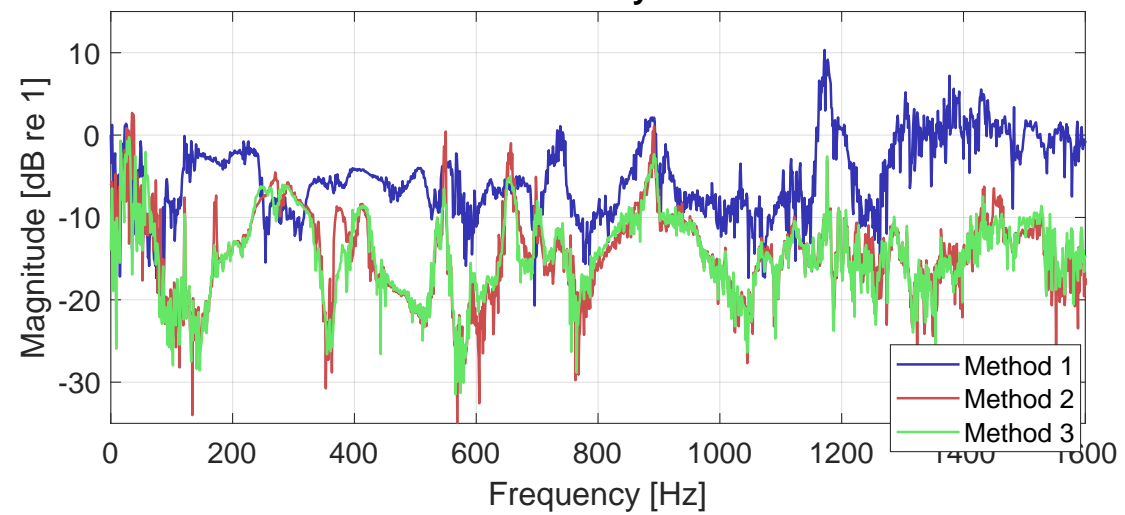

Figure 8: Element of the TM: receiver position $R_{3}$, path 3

Lastly, the individual contributions for all three OTPA-based methods are,

$$
S_{i j}=T_{i j} V_{j}, \quad Y_{i}=\sum_{j} S_{i j}
$$

195 and the corresponding indicators responses.

\section{Results \& Analysis}

The individual contributions estimated with three OTPA-based methods (Method 1, 2, 3) are compared with the baseline: TPA, referred to as Exact.

the differences between the methods are the different transmissibility matrices For the analytical model, Exact represents actual individual contributions, but this is not the case for the experimental setup, due to missing rotational DoF. However, for the experimental setup, it is assumed that the effect of rotational DoF is minimal and can be neglected; the result in figure 7 supports that.

In order to compare many individual contributions and how well the results of each OTPA-based methods matched TPA experimentally, Frequency Response Assurance Criteria (FRAC) were employed. The FRAC varies from 0 to 1 , where 1 represents the perfect match between two spectra [20]. The FRAC values for receivers positions $R_{3}$ and $R_{4}$, which measures in $Z$ and $Y$ directions, are presented in figures $9 \mathrm{a}$ and $9 \mathrm{~b}$ together with the mean values and 10 the standard deviation. 


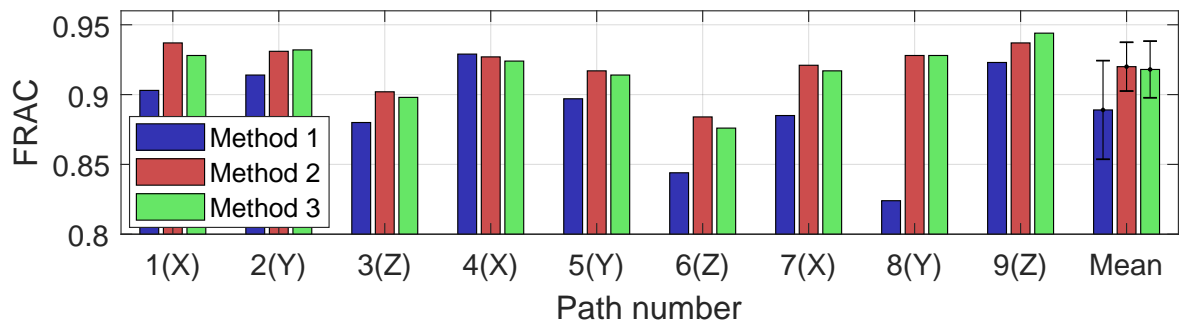

(a) Receiver R3

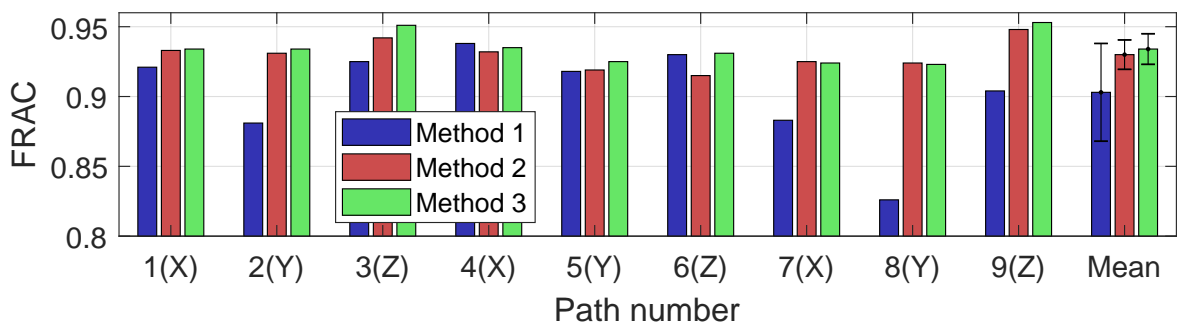

(b) Receiver R4

Figure 9: FRAC value

From figure $9 \mathrm{a}$ it can be noted that for receiver position $R_{3}$, Method 2 matched the best to TPA method, since it has the highest FRAC values for most of the paths, closely following by Method 3 and then Method 1. Regarding figure 9b it can be observed that for the $R_{4}$ position, the highest FRAC values 215 are obtained with Method 3, following by Method 2 and Method 1. The FRAC results from figure 9 and the receivers' total average values were checked using the ANOVA model, which are presented in table 4. It can be observed

\begin{tabular}{c|c|c|c} 
& $R_{3}$ & $R_{4}$ & Receivers average \\
\hline Methods & $p$-value & $p$-value & $p$-value \\
\hline $1 \& 2$ & 0.03 & 0.04 & 0.03 \\
$1 \& 3$ & 0.047 & 0.02 & 0.01 \\
$2 \& 3$ & 0.77 & 0.38 & 0.68
\end{tabular}

Table 4: ANOVA: receiver position $R_{4}$ 
that the results from Method 1 are indeed significantly different from Method 2 and Method 3; the $p$-value is below $5 \%$.

The individual contributions of transverse paths $(Z)$ of receiver position $R_{3}$ are presented in figure 10

From the figure 10a it can be observed that all three OTPA-based methods match TPA rather well over the full frequency range, where the biggest differences appear in the $200-400 \mathrm{~Hz}$ range. According to figure 9a, for path 3 , Method 2 matched the best with TPA following by Method 3 and Method 1.

Regarding figure 10b, it can be seen that each OTPA-based method shows an inferior match to TPA compared with previous plots. It can be observed that Method 2 and Method 3 match TPA quite well in the range of $1-200$ $\mathrm{Hz}$, and the biggest differences appear at around $400-800 \mathrm{~Hz}$. The individual contribution estimated with Method 1 shows more discrepancy to TPA, hence the FRAC value was lower.

Lastly, from the figure $10 \mathrm{c}$ it can be noted that all three OTPA-based methods show a rather good match to TPA. The best match is achieved with Method 3, following by Method 2 and Method 1. From the plots in the figure 10, it can be clearly noticed that Method 3 and Method 2 coincide better to TPA comparing with Method 1.

The individual contribution plots of receiver position $R_{4}$, estimated in the same directions $Y$, are presented in figure 11 .

From figure 11a it can be observed that all three OTPA-based methods match TPA quite well in the frequency range of $100-600 \mathrm{~Hz}$, but above that, they become more different, especially Method 1. Also, it can be noted that individual contributions estimated with Method 2 and Method 3 are quite similar, which was also previously observed.

245 In figure $11 \mathrm{~b}$ all three OTPA-based methods show rather similar behavior between each other and also match TPA quite well. The biggest differences between TPA and OTPA-based techniques appear at the frequency range of $600-1000 \mathrm{~Hz}$, while at other frequencies only minor ones can be observed. 


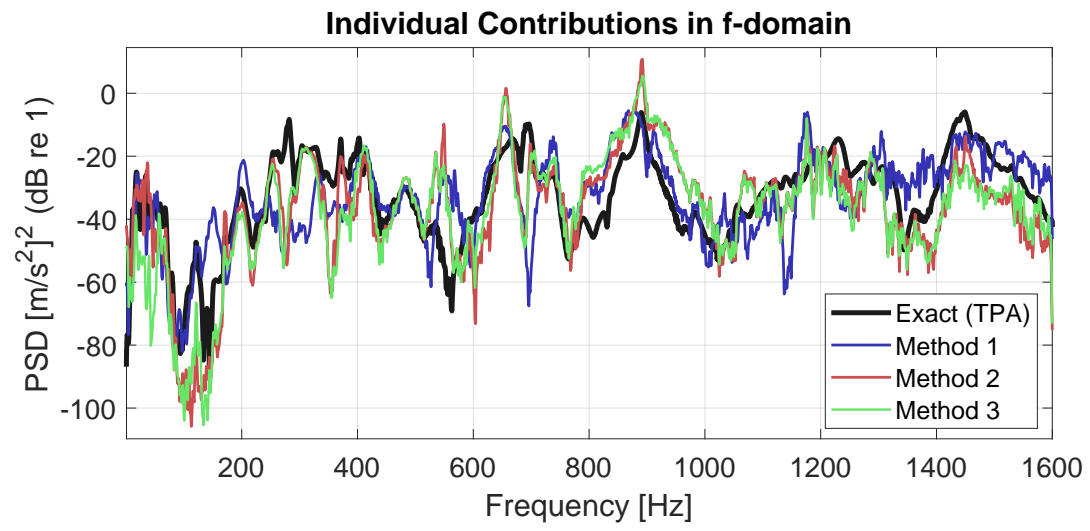

(a) Path 3

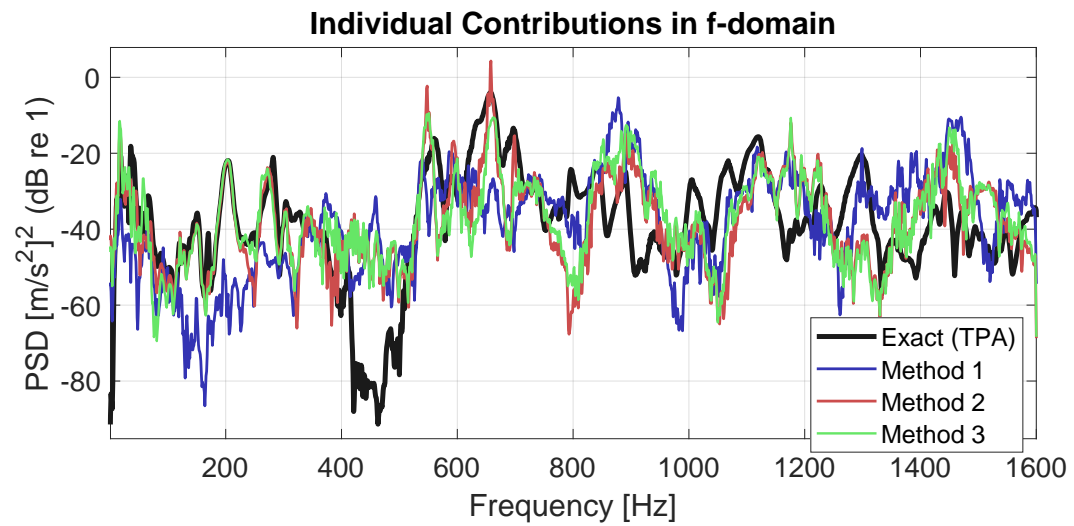

(b) Path 6

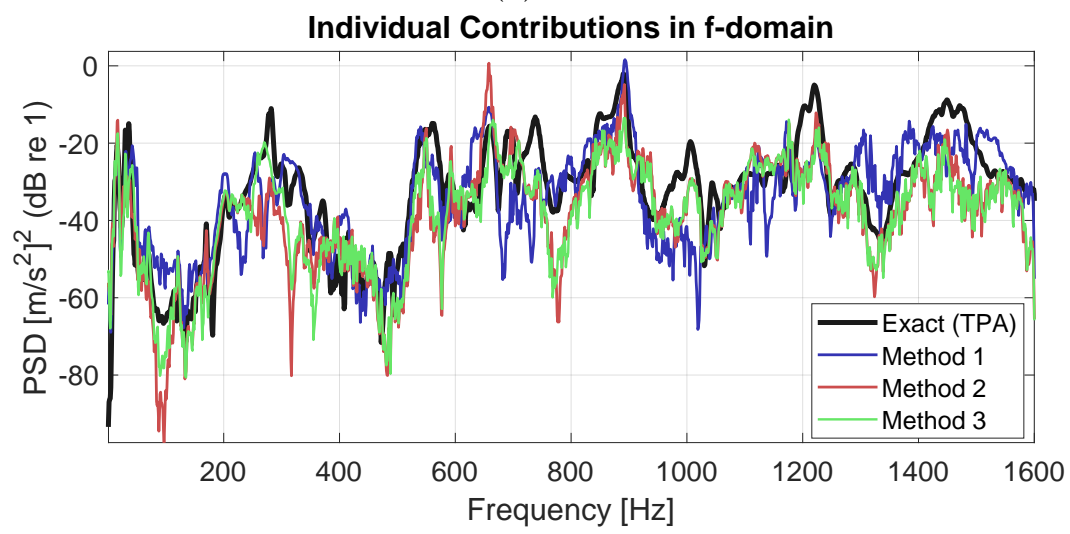

(c) Path 9

Figure 10: Individual contributions at $R_{3}$ position 


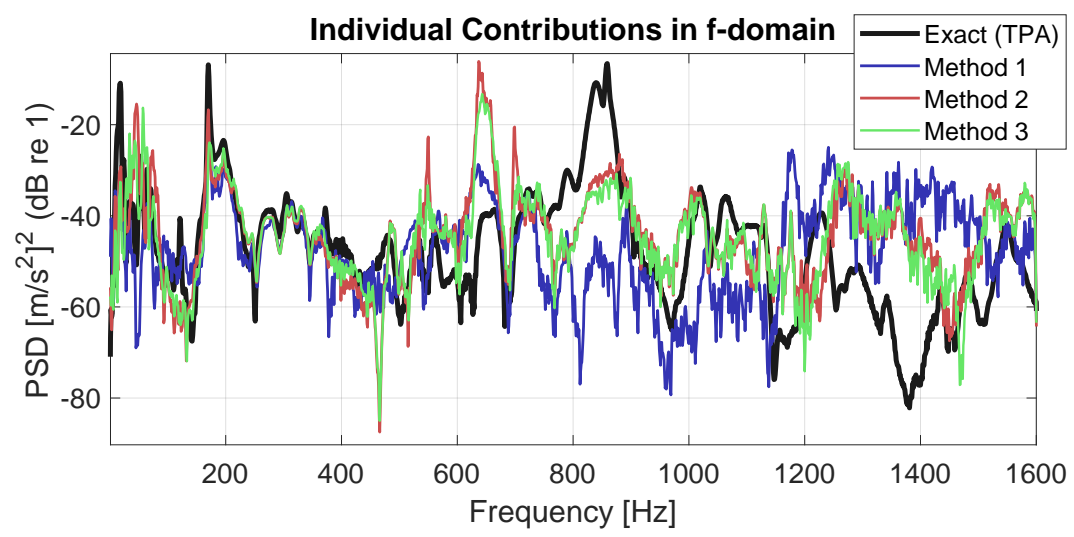

(a) Path 2

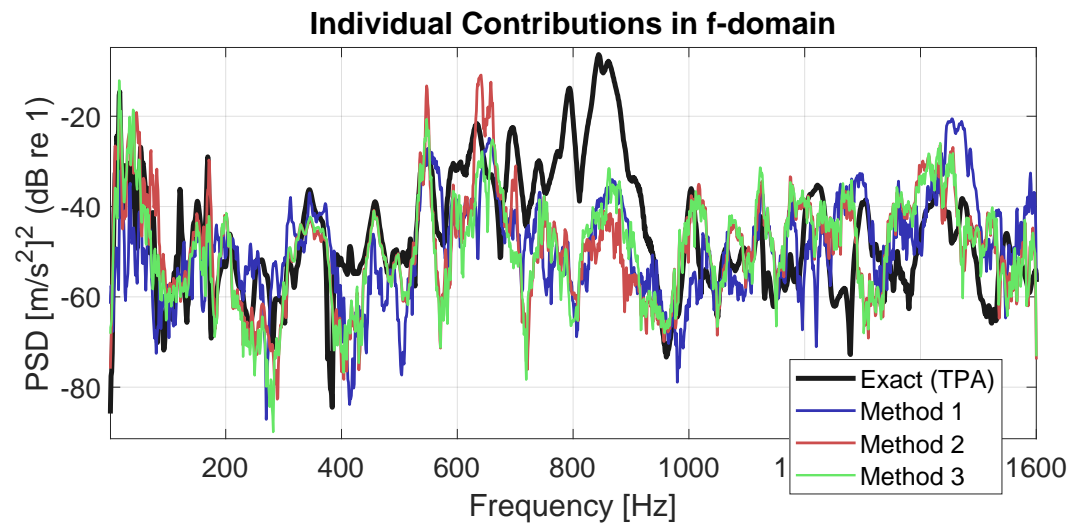

(b) Path 5

Individual Contributions in f-domain

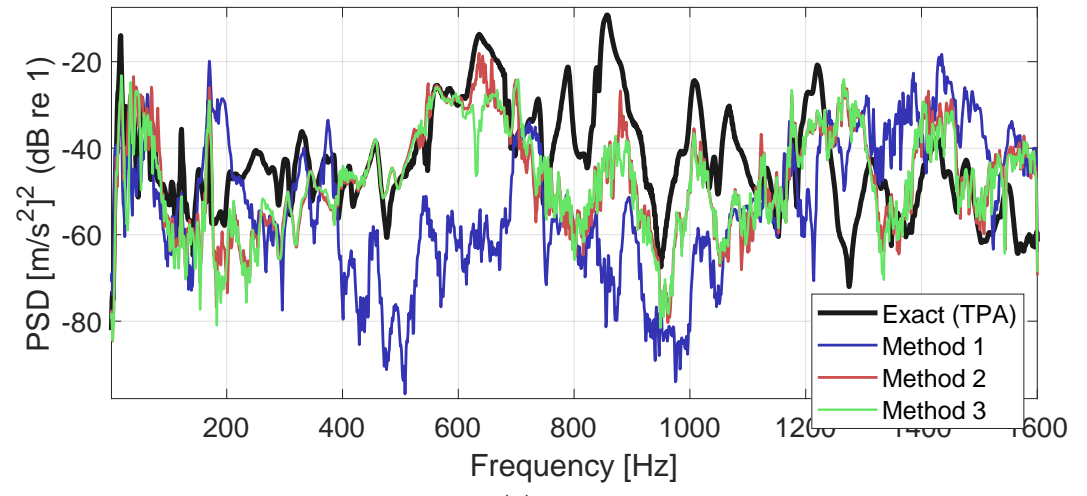

(c) Path 8

Figure 11: Individual contributions at $R_{4}$ position 
The individual contributions plot in figure $11 \mathrm{c}$ shows that Method 2 and

$\mathbf{1}$, that was also observed from figure $9 \mathrm{~b}$.

The plot of individual contributions containing all nine paths for receiver position $R_{3}$ is presented in figure 12 It is observed that the best matches to TPA come from Method 2 and Method 3 at frequencies of $10-600 \mathrm{~Hz}$, also above $1000 \mathrm{~Hz}$.
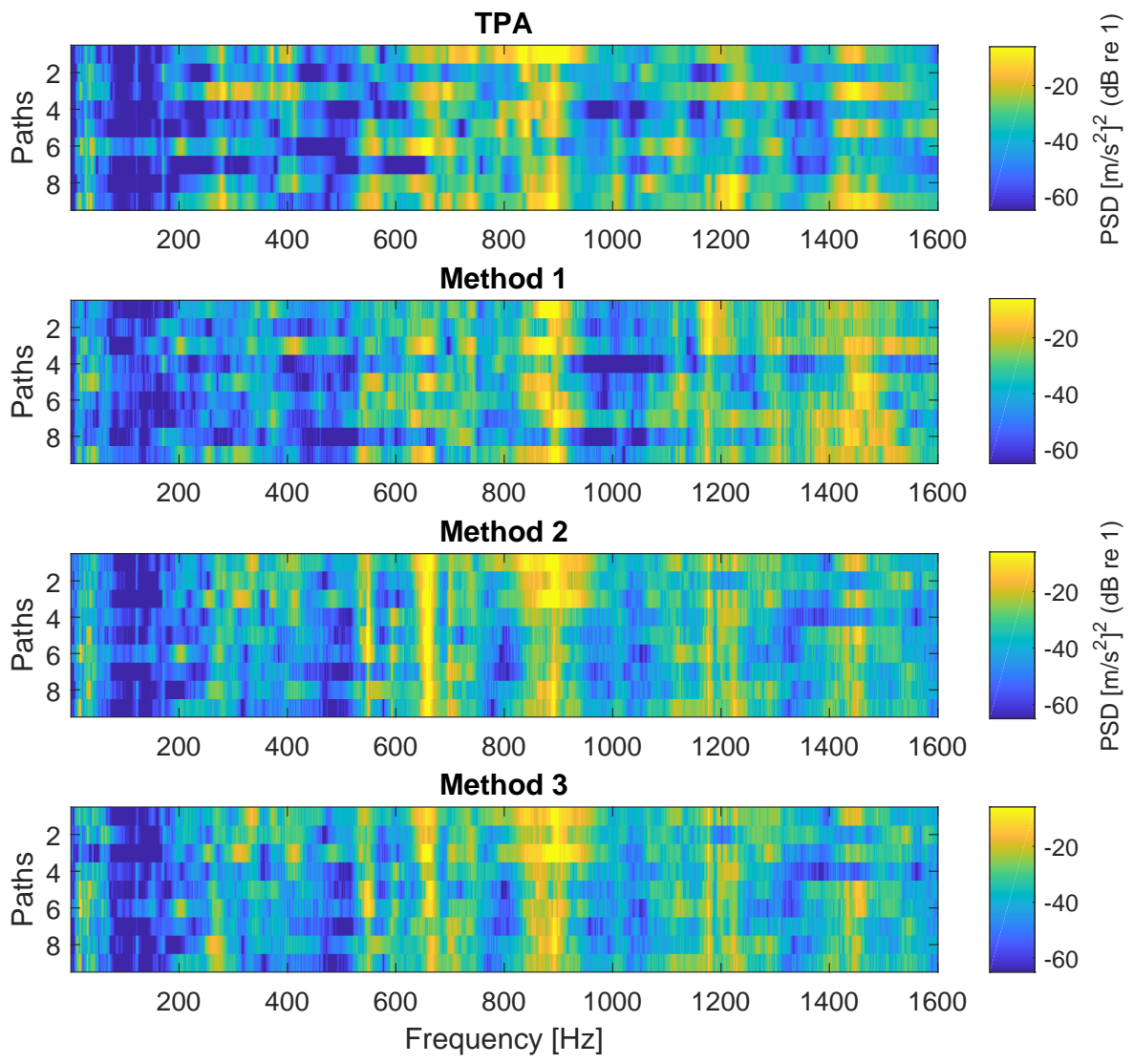

Figure 12: Individual contributions at $R_{3}$ position

From figures 9a, 9b and the individual contribution plots it was noted that the best results were achieved with Method 2 and Method 3, while Method 1 showed bigger differences with TPA. In addition to that, the results achieved 
with Method 2 and Method 3 were rather similar, which was also observed

produced by Method 2 and Method 3 become quite similar.

Also, from the figures 9a, 9b and individual contribution plots, it was noted that from all three OTPA-based methods, Method 1 matched the worst with TPA. These results suggest that individual contributions estimated with Method have any influence. Indeed, from the FRF plots it was observed that by exciting one path, the responses in other paths are quite significant due to cross-talk, hence it makes sense that Method 1 results showed the lowest consistency with TPA. Regarding the Method 2, by analyzing the analytical model, it was parameter. By having the soft engine mounts, the cross-coupling between the paths is highly reduced, which results in more correct individual contributions.

\section{Discussion}

From the previous section, it was observed that the results of Method 2 and Method 3 matched the best with TPA. However, according to the analytical model, it was expected that Method 3 would agree with TPA almost perfectly, which was not achieved experimentally.

Having produced and analyzed the obtained results, it is learned that the chosen experimental setup was not perfect for studying OTPA-based methods. drawbacks concerning different methods are listed below:

- Considering the TPA results, it was noted that all the paths contribute 
rather similarly. First, all the forces were highly correlated, which could be reduced by having three separate sources for each mount. By having three separate sources, it would be possible to excite three different frequency ranges, for example, mount 1: $0-500 \mathrm{~Hz}$, mount 2: $500-1000 \mathrm{~Hz}$ and mount 3: $1-1.5 \mathrm{kHz}$, similarly to [16]. This will allow investigating the cross-coupling effect for the OTPA-D method, which theoretically is supposed to be zero.

295

- Second, in order to differentiate individual contributions between each path, the design of the frame should be changed. Considering the current frame, the FRFs between left and right mounts are rather similar due to the same structure and since the forces are highly correlated, the contributions between the left and right sides become quite alike. By changing the structure on one side, or both, the FRFs will become more different, causing difference in contributions, which will give a better identification of the most dominant paths determined by each method.

- Installing harder engine mounts shall increase the cross-coupling effect in the active part. Due to stiffer interfaces, Method 2 results shall regress, while for Method 3 they shall not, which will better represent the analytical model from [9].

- Missing rotational coupling, which cannot be identified with TPA method. In many practical cases, rotational DoFs are assumed to be insignificant and hence neglected. However, the level of rotational DoF cannot be identified and therefore TPA results have to be trusted as they are. It was shown that the measured response matched the sum of individual contributions very well, only with small discrepancies, see figure 7. That suggests that rotational coupling was insignificant in this experiment. However, it would be interesting to investigate the rotational coupling effect to find out the actual influence.

- From the analytical model, it follows that Method 3 does not address the 
cross-coupling issue, and considering that all the structure-borne paths were included, it can be suggested that the issue appears to be due to imperfect TM estimation. The acting forces and operational conditions during the experiments were rather similar, which might have caused illconditioning and therefore TM estimation became somewhat incorrect. It is expected that due to this reason, the results achieved with OTPAD method were not fully consistent to TPA. In addition to that, this issue might have also influenced the results obtained with Method 1 and Method 2.

- In this experiment, Signal to Noise Ratio (SNR) was considered to be high and hence not investigated. However, in a future research, it would be interesting to investigate SNR as well. By knowing that engine mounts were rather soft, the SNR is more likely to be higher at the active side than at the passive one. If SNR ratio is low, the estimated TMs become partially incorrect and hence the final individual contributions. As the result, the individual contributions, estimated with Method 1 and Method 3, become more unreliable than Method 2, as well as different from TPA results.

Overall, it is shown that Method 1 gives the worst results. Also, Method 2 results showed that OTPA are quite reliable if the indicators are placed on the active side and the mounts are rather soft. Summarizing, to determine quite realistic individual path contributions, the OTPA-D method can be applied, since it produces a rather trustworthy outcome and has a significantly faster procedure than conventional TPA. If the mounts are known to be soft, the individual contributions can be determined by the OTPA method using active side responses, but if the mounts are stiff or their stiffnesses are unknown, it is recommended to use the OTPA-D method. 


\section{Conclusion}

345 were compared analytically and experimentally. From the experimental results, it was noted that the results obtained with Method 2 and Method $\mathbf{3}$ were rather similar due to soft engine mounts. Also, both Method 2 and Method 3 showed a better consistency to TPA compared with Method 1. However, of OTPA and OTPA-D very well, hence the additional suggestions regarding the improvement of experimental setup were presented.

The main drawback the OTPA-D method faces is the number of indicators accelerometers used, which is twice the amount required for traditional OTPA. Also, since OTPA-D is based on decomposition principle, the method suffers the issues described in [2, 4], that are well-know problems regarding the OTPA approach. Nevertheless, it is suggested to use the OTPA-D method over OTPA if the additional amount of accelerometers are available. Also, the experimental results showed that the OTPA technique produces results close to OTPA-D that

are also similar to TPA if the active side indicators responses and soft engine mount are used. To sum up, even though the OTPA-D technique did not perfectly match TPA results, it showed an improvement towards TPA estimation regarding the individual contributions and a lot of potential for a future investigation. In addition to that, the procedure of OTPA-D technique is significantly

faster than conventional TPA, which is a big advantage for industries unwilling to spend extra time conducting TPA measurements.

\section{References}

[1] der Seijs MVV, de Klerk D, Rixen DJ. General framework for transfer path analysis: History, theory and classification of techniques. Mechanical Systems and Signal Processing 2016;68-69:217

घ -44. URL: http://wwW.sciencedirect.com/science/article/pii/ 
S0888327015003647, doi https://doi.org/10.1016/j.ymssp.2015.08. 004 .

[2] Gajdatsy P, Janssens K, Gielen L, Mas P, Auweraer HVD. Critical assesment of operation path analysis: effect of neglected paths. Proceedings of the XV International Congresson Sound and Vibration (ICSV), Daejeon, Korea 2008;:1090.

[3] Gajdatsy P, Janssens K, Gielen L, Mas P, Van Der Auweraer H. Critical assessment of operational path analysis: effect of coupling between path inputs. The Journal of the Acoustical Society of America 2008;123(5):3876. URL: https://doi.org/10.1121/1.2935777, doi:10. 1121/1.2935777, arXiv:https://doi.org/10.1121/1.2935777.

[4] Gajdatsy P, Janssens K, Gielen L, Mas P, Van Der Auweraer H. Critical assessment of operational path analysis: mathematical problems of transmissibility estimation. The Journal of the Acoustical Society of America 2008;123(5):3869. URL: https://doi.org/10.1121/1.2935749 doi:10.1121/1.2935749, arXiv:https://doi.org/10.1121/1.2935749.

[5] Janssens K, Gajdatsy P, Gielen L, Mas P, Britte L, Desmet W, et al. Opax: A new transfer path analysis method based on parametric load models. Mechanical Systems and Signal Processing 2011;25:1321-38.

[6] Moorhouse A, Elliott A, Evans T. In situ measurement of the blocked force of structure-borne sound sources. Journal of Sound 口 and Vibration 2009;325(4):679-85. URL: http://www.sciencedirect. 口 com/science/article/pii/S0022460X09003794, doi:https://doi.org/ $10.1016 / j \cdot j s v .2009 .04 .035$

[7] Shin T, Kim YS, An K, Lee SK. Transfer path analysis of rumbling noise in a passenger car based on in-situ blocked force measurement.

(1) Applied Acoustics 2019;149:1-14. URL: http://www.sciencedirect.

घ com/science/article/pii/S0003682X18308569, doi/https://doi.org/ 400 $10.1016 / \mathrm{j}$. apacoust. 2019.01 .015 
[8] Diez A, Battarra M, Palenzuela J, Cervantes G, Walsh S, De-la Cruz M, et al. Comparison between transfer path analysis methods on an electric vehicle. Applied Acoustics 2017;118:83-101. doi:10.1016/j.apacoust. 2016.11 .015

[9] Tcherniak D. Application of transmissibility matrix method to structure borne path contribution analysis. Proceedings of NAG/DAGA, Rotterdam, Holland 2009;:1691-4.

[10] Plunt J. Finding and fixing vehicle NVH problems with transfer 1 path analysis. Sound and Vibration 2005;39(11):12-6. URL: https: //www. scopus. com/inward/record.uri?eid=2-s2.0-34547237835\& partnerID=40\&md5=b77e39ba1962e10553f70ac9a29f bc23

[11] Thompson D, van Vliet W, Verheij J. Developments of the indirect method for measuring the high frequency dynamic stiffness of resilient elements. Journal of Sound and Vibration 1998;213(1):16988. URL: http://www.sciencedirect.com/science/article/pii/ S0022460X98914924, doi/https://doi.org/10.1006/jsvi.1998.1492.

[12] Dobson BJ, Rider E. A review of the indirect calculation of excitation forces from measured structural response data. Proceedings of the Institution of Mechanical Engineers, Part C: Mechanical Engineering Science 1990;204(2):69-75. URL: https://doi.org/10.1243/PIME_PROC_1990_ 204_080_02, doi:10.1243/PIME\_PROC\_1990\_204\_080\_02.

[13] Ben-Israel A, Greville TNE. Generalized Inverses: Theory and Applications. New York, NY: Springer; 2002. ISBN 978-0-387-00293-4. doi $10.1007 /$ b97366

425 [14] Zhang Y. Future Communication, Computing, Control and Manage口 ment: Volume 2; vol. 142. 2012. ISBN 978-3-642-27313-1. doi 10.1007/ 978-3-642-27314-8. 
[15] Maia N, Silva J, Ribeiro A. The transmissibility concept in multi-degree-of-freedom systems. Mechanical Systems and Signal Processing 2001;15(1):129-37. URL: http://www.sciencedirect. 1. com/science/article/pii/S0888327000913566 doi/https://doi.org/ $10.1006 / \mathrm{mssp} .2000 .1356$

[16] Tcherniak D, Schuhmacher A. Application of transmissibility matrix method to NVH source contribution analysis. Proceedings of IMAC, Orlando (FL), USA 2009;:2076-86.

[17] der Auweraer HV, Mas P, Dom S, Vecchio A, Janssens K, de Ponseele PV. Transfer Path Analysis in the Critical Path of Vehicle Refinement: The Role of Fast, Hybrid and Operational Path Analysis. SAE International; 2007. URL: https://doi.org/10.4271/2007-01-2352 doi $10.4271 / 2007-01-2352$.

[18] Leclere Q, Roozen B, Sandier C. On the use of the Hs estimator for the experimental assessment of transmissibility matrices. Mechanical Systems and Signal Processing 2014;43(1-2):237-45. URL: https://hal. archives-ouvertes.fr/hal-00911916

[19] de Klerk D, Ossipov A. Operational transfer path analysis: Theory, guidelines and tire noise application. Mechanical Systems and Sign nal Processing 2010;24(7):1950-62. URL: http://www.sciencedirect. a com/science/article/pii/S0888327010001524. doi/https://doi.org/ 10.1016/j.ymssp.2010.05.009 special Issue: ISMA 2010.

450 [20] Ewins DJ. Modal testing: theory, practice, and application. 2nd ed ed.; Baldock, Hertfordshire, England ; Philadelphia, PA : Research Studies Press; 2000. ISBN 0471975184 (Wiley : hardback). Previous ed.: 1995. 